\title{
Synthesis and Applications of Two-Dimensional Transition Metal Sulfide Nanomaterials in Photothermal Therapy
}

\author{
Wentao Cao, ${ }^{a}$ Rui Liu, ${ }^{*, c}$ and Jun Zhu ${ }^{\star, b, c}$ \\ a School of Medical Instrument and Food Engineering, University of Shanghai for Science and Technology, \\ 516 Jun Gong Road, Shanghai 200093, China \\ ${ }^{b}$ Shanghai University of Medicine \& Health Sciences, 279 Zhou Zhu Highway, Shanghai 201318, China \\ ${ }^{c}$ National Engineering Research Center for Nanotechnology, 28 East Jiang Chuan Road, \\ Shanghai 200241, China
}

Email: nercn_liurui@163.com (R. L.), yzjzhu@163.com (J. Z.)

\begin{abstract}
Recently, cancer has becomes one of the best serious diseases which threatens seriously the life and health of human. Although the radiotherapy and chemotherapy have been used to treat cancer, these traditional treatments have always their own limitations. Therefore, a few materials and strategies have mentioned, many of which focused their attention on the two-dimensional (2D) photothermal nanomaterials including graphene, transition metal sulfide, and so on. There are good electronic transmission performances in these 2D nanomaterials, which contribute to the prefect photothermal conversion for cancer treatments. Additionally, their larger specific surface area can be used to load the drugs or integrate with other functional nanomaterials, and they with modified surface can be stabilized in organism and congregated in tumor site through an enhanced permeability and retention effect (EPR) effect. In order to understand the 2D nanomaterials deeply, we review some typical 2D transition metal sulfide nanomaterials including their preparation, modification and applications in biology.
\end{abstract}

Keywords 2D nanomaterials, transition metal sulfide, cancer, photothermal therapy

\section{Introduction}

The two-dimensional (2D) nanomaterials' arrangement of atoms and bond forces is similar to but stronger than that of three-dimensional (3D) nanomaterials. For example, graphene is considered as the most classical $2 \mathrm{D}$ nanomaterial. It is a single layer for the 2D honeycomb hexagonal planar crystal composed of the hybrid carbon atoms and the basic component unit of the carbon nanomaterials. Additionally, the transition metal sulfide considered as the inorganic graphene has a layered structure, which is similar to that of graphene. The layers are connected with the weak van der Waals forces, and each layer is connected with the covalent bind between the transition metal and sulfur elements. These special structures endow the $2 \mathrm{D}$ nanomaterials to have many strange and useful properties such as huge specific surface area, special electronic structure, and good mechanics performance, ${ }^{[1]}$ which has been developed rapidly in some fields such as energy conversion and cancer treatment. ${ }^{[2]}$ To these days, more and more $2 \mathrm{D}$ nanomaterials have been obtained and applied, including hexagonal boron nitride ${ }^{[3]}$ transition metal chalcogenide ${ }^{[4]}$ transition metal oxide ${ }^{[5]}$ transition metal carbides or carbonitride, ${ }^{[6]}$ black phosphate, ${ }^{[7]}$ and so on.

As a new class of 2D nanomaterial, transition metal sulfide has received tremendous attention and showed great promise in various areas including biomedicine. Recently, several classes of high-performance nanomaterials have been reported along with significant progress in understanding their physical properties. Thus, we present a review of their architecture, operating principles and methods, and photothermal therapy.
By critically assessing and comparing the performance of these nanomaterials, some synthetic techniques and applied fields based on 2D transition metal sulfide nanomaterials are characterized and identified. This work may offer new opportunities to $2 \mathrm{D}$ transition metal sulfide nanomaterials for clinical applications.

\section{Structure and properties of 2D transition metal sulfide}

The 2D transition metal sulfide refers to the compound with the transition metal group elements $(\mathrm{M})$ and the sulfur group non-metallic elements $(X)$, which constructs the $\mathrm{X}-\mathrm{M}-\mathrm{X}$ sandwich structure (Figure 1 ). The 2D transition metal sulfide nanomaterials are a layered structure and composed of the three layers of atoms. The neighboring X-M atoms are connected by the covalent bonds. At the same time, there is weak van der Waals force between layer and layer. ${ }^{[8,9]}$ Based on such structural characteristics, these nanomaterials have many special properties, such as the large specific surface area, the high near infrared absorption, and the large atomic number, which has made them develop rapidly in the biomedical aspects such as bio-detection, bio-imaging and tumor treatment. ${ }^{[10]}$

\section{Synthesis of 2D transition metal sulfide nano- materials}

The preparation methods of the $2 \mathrm{D}$ transition metal sulfide nanomaterials can be divided into the top-down exfoliation method and the bottom-up synthesis method. The top-down 


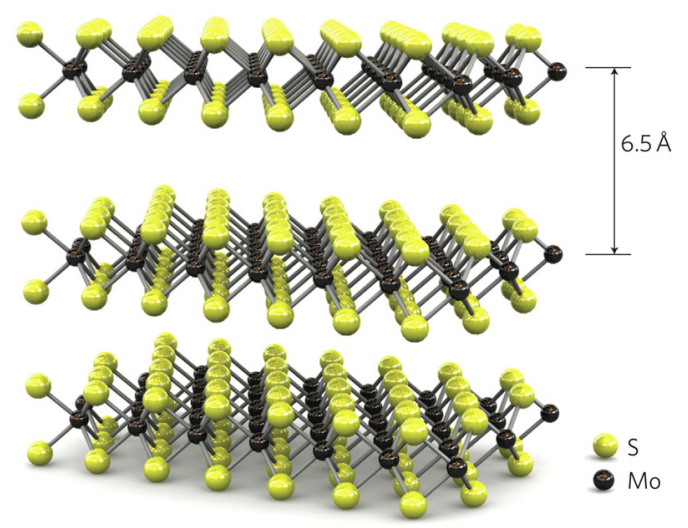

Figure 1 Three dimensional structure of $\mathrm{MoS}_{2}$. Single layers, $6.5 \AA$ thick, can be extracted using scotch tape-based micromechanical cleavage. ${ }^{[11]}$

and bottom-up approach means that researchers break the bulk materials down into smaller structures by the chemical or physical methods, and use the chemical methods from atom to nanomaterials, respectively. The general physical method is rigorous for the equipment and reaction conditions, while the general chemical method is simple and flexible. Specially, these methods can be basically divided into the six kinds, including mechanical exfoliation, chemical vapor deposition (CVD), colloidal synthesis, hydrothermal synthesis, the intercalation exfoliation, and the liquid phase exfoliation. The CVD, colloidal and hydrothermal methods belong to the bottom-up modes, while others belong to the top-down preparation method.

\section{Mechanical exfoliation}

The mechanical exfoliation is a traditional and mature preparation method, which is famous for relatively simple operation and the high degree of exfoliation. Geim et al. used this method to discover graphene in 2004. Later, Geim et al. also used this method to synthesize the single-layer $\mathrm{NbSe}_{2}, \mathrm{BN}$, $\mathrm{MoS}_{2}, \mathrm{WS}_{2}$, and so on. ${ }^{[12,13]}$ This method has been regarded as one of the most classical methods to prepare the 2D nanomaterials. The basic principle of this method is that the additional adhesion strength is more than the interlaminar van der Waals forces of the transition metal sulfide, which leads to strip the lamellar transition metal sulfide to get the 2D nano- sheets. There are many advantages of the mechanical exfoliation, including an excellent quality, a simple process, a low cost, and so on. However, it is unsuitable for the large scale production because of its poor repeatability. Thus, this method is commonly used to synthesize the 2D nanomaterials in laboratory and difficult to be applied in industry.

\section{Intercalation exfoliation}

The intercalation-exfoliation method uses small molecules to insert the interlamination in order to obtain the interlaminar compounds. As a result, the interlaminar forces are reduced. The laminar materials are stripped easily to obtain the $2 \mathrm{D}$ nanomaterials. The intercalation reagents include mainly $\mathrm{H}_{2} \mathrm{SO}_{4}$ and $\mathrm{HNO}_{3}$ with the poor thermal stability and alkali metal with the poor chemical stability such as $\mathrm{Li}, \mathrm{K}, \mathrm{Cs}, \mathrm{Rb}$, in which $\mathrm{Li}$ ion intercalation is most commonly used. For example, Zeng et al. used the electrochemical lithium intercalation method to prepare a series of the single-layer 2D TMD nanomaterials such as $\mathrm{MoS}_{2}, \mathrm{TaS}_{2}, \mathrm{TiS}_{2}, \mathrm{WS}_{2}$, and $\mathrm{ZrS}_{2}{ }^{[14]}$ Yang et al. used $n$-butyllithium to intercalate $W_{2}$ powder in order to synthesize $W_{2}$ nanosheets. ${ }^{[15]}$ The intercalation exfoliation method is easy to operate and could produce the $2 \mathrm{D}$ nanomaterials on a large scale. However, its drawback is the long reaction time and the high reaction temperature.

\section{Liquid phase exfoliation}

The liquid phase exfoliation method means that a small amount of the powder solid is dissolved into the solvent forming the low concentration of the dispersion liquid, and ultrasound is used to destroy the interlaminar van der Waals forces. As a result, a large number of the homogeneous multilayer or even single-layer nanofilm solution can be obtained. The method is generally divided into two types including organic solvent exfoliation method and aqueous surfactant method. Coleman et al. used 1-Methyl-2-pyrrolidinone (NMP) as the reaction solvent to prepare $\mathrm{MoS}_{2}, \mathrm{WS}_{2}, \mathrm{MoSe}_{2}, \mathrm{MoTe}_{2}, \mathrm{TaSe}_{2}$, and other 2D nanomaterials. ${ }^{[16]}$ Smith et al. used water as solvent directly and added the surfactant forming water-surfactant system to inhibit the product agglomeration. ${ }^{[17]}$ As a result, they synthesized many kinds of transition metal sulfide and boron nitride (BN) nanomaterials. Although the liquid phase exfoliation has realized the batch preparation of $2 \mathrm{D}$ transition metal sulfide nanomaterials, the method has a low efficiency for exfoliation.

\section{Chemical vapor deposition}

The chemical vapor deposition is that the reagents with required elements are vaporized, decomposed, resynthesized and deposited at high temperature by means of the thermal decomposition and chemical synthesis. It is a commonly used for preparing the inorganic nanomaterials in industry. For example, the tungsten source is deposited on the substrate surface and then vulcanized or thermally decomposed at high temperature. Specially, $\mathrm{WO}_{3}$ as the precursors was deposited to $\mathrm{WO}_{x}$ on the basis for $\mathrm{Si} / \mathrm{SiO}_{2}$ through the predeposition step, and then $S$ steam was vulcanized and coated on $\mathrm{WO}_{x}$ directly. At last, the $\mathrm{WS}_{2}$ nanosheet was obtained. ${ }^{[18]}$ Additionally, the tungsten source was sublimed completely at high temperature, reacted with the sulfur vapor to form $W S_{2}$ and then $W_{2}$ is deposited on the surface of substrate. This process is a controllable gas phase and can also prepare a monolayer or small layer $\mathrm{MoS}_{2}$ film with the large area and the high crystallinity. ${ }^{[19]}$ This method can be used to prepare a large number and high quality $2 \mathrm{D}$ nanofilms with the controllable thickness, the good harmonic, and the electrical property. ${ }^{[20]}$ However, the CVD preparation conditions are harsh and the applicability is relatively narrow, which is restricted for many $2 \mathrm{D}$ transition metal sulfide nanomaterials except for $\mathrm{MoS}_{2}, \mathrm{WS}_{2}, \mathrm{MoSe}_{2}$, and so on. ${ }^{[21]}$

\section{Colloidal synthesis}

Some surface modifiers or reductants are added to the solvent at the high boiling point and pyrolyzed the precursors so as to obtain the inorganic nanocrystals with the uniform size. For example, Seo et al. synthesized a 2D WS ${ }_{2}$ nanofilm in 2007. Firstly, the tungsten oxide nanorods $\left(\mathrm{W}_{18} \mathrm{O}_{49}\right)$ reacted with hexadecyl amine under the argon gas protection. ${ }^{[22]}$ Then they were heated and reacted with injected $\mathrm{CS}_{2}$ under the vacuum. At last, the single layer or multilayer nano layered $\mathrm{WS}_{2}$ crystal was gradually formed. This method is also used to synthesize $\mathrm{MoS}_{2}, \mathrm{GeS}_{2}, \mathrm{TiS}_{2}$ and other monolayer or multilayer nanometer thin films. Additionally, Jeong et al. used the method to synthesize a series of the $2 \mathrm{D}$ lamellar materials such as $\mathrm{TiS}_{2}, \mathrm{ZrS}_{2}$, $\mathrm{HfS}_{2}, \mathrm{VS}_{2}, \mathrm{NbS}_{2}, \mathrm{TaS}_{2}, \mathrm{TiSe}_{2}$ and $\mathrm{ZrSe}_{3}$ in 2012. ${ }^{[23]}$ The advantages of the colloidal synthesis method lies in the simple process, which can be realized easily in the large-scale production, while the disadvantage is that its controllability and repeatability is poor. 


\section{General Chemistry}

\section{Hydrothermal synthesis}

The hydrothermal synthesis method means that water or solvent as the reaction medium dissolves the indissolvable or insoluble substances and conducts the recrystallization reaction at high temperature and high pressure environment. The reaction temperature and pressure is generally from 100 to $1000^{\circ} \mathrm{C}$ and from $1 \mathrm{MPa}$ to $1 \mathrm{GPa}$, respectively. It is a promising method for synthesis of the transition metal sulfide nanomaterials because of its simple operation, low cost and high purity. For example, Wang et al. used the sodium moly- bdate and cysteine as precursors and synthesized $\mathrm{MoS}_{2}$ quantum dots by heating them for $36 \mathrm{~h}$ at $200{ }^{\circ} \mathrm{C} .{ }^{[24]}$ Ren et al. reported a simple hydrothermal method to synthesize the $\mathrm{MoS}_{2}$ quantum dots. ${ }^{[25]}$ They further used the sodium molybdate as the sources of molybdenum and used dibenzyl disulfide as the sources of sulfur and synthesized a single-layer $\mathrm{MoS}_{2}$ quantum dot with thickness about $3.6 \mathrm{~nm}$ at $220{ }^{\circ} \mathrm{C}$. Liu et al. presented the preparation of stable $1 \mathrm{~T}-\mathrm{WS}_{2}$ ultrathin nanosheets with $\mathrm{NH}^{4+}$ intercalation by a bottom-up hydrothermal method. ${ }^{[26]}$ The nanosheets have a good hydrophilic, strong light absorption and excellent photostability in the broad IR wavelength region.

\section{Application of 2D transition metal sulfide nano- materials in cancer diagnosis and treatment}

Recently, it is interesting to design a multifunctional nanoprobe for combined imaging, because each imaging modality has its own limitations and advantages. For instance, numerous nanoprobes used in luminescent imaging, computed tomography $(\mathrm{CT})$, ultrasound imaging and magnetic resonance imaging (MRI) have been applied for cancer diagnosis to improve sensitivity and accuracy. Additionally, the traditional methods for cancer treating include mainly chemotherapy and radiotherapy. However, they also damage the normal tissues limiting the further development. In order to treat tumor more effectively, 2D transition metal sulfide nanomaterials with the good IR absorption and photothermal conversion capability are used to treat tumors. Furthermore, in order to enhance the hydrophilicity and biocompatibility of the $2 \mathrm{D}$ transition metal sulfide nanomaterials, the researchers use the functional polymers to modify their surface so that they have a good stability in the physiological environment. ${ }^{[15]}$

\section{Photoacoustic and multimodal imaging}

The photoacoustic imaging is a new method for medical image. ${ }^{[27]}$ The imaging uses the light absorption of organization or the developer to convert the pulsed laser into the heat energy, which induces the thermal expansion producing ultrasonic and then detect the ultrasonic signals to reconstruct image. 2D transition metal sulfide nanomaterials have a high light absorption capacity in the whole IR region and are an ideal photoacoustic imaging agents. Qian et al. used the IR light absorption and photothermal transformation properties of 2D $\mathrm{TiS}_{2}$ nanosheets to conduct the photoacoustic imaging. ${ }^{[28]}$ Most of the blood vessels and tissues of the tumor can be clearly seen after injection of $\mathrm{TiS}_{2}-\mathrm{PEG}$ although only major blood vessels could be seen in the tumor before the injection of $\mathrm{TiS}_{2}-\mathrm{PEG}$.

Compared to the traditional optical imaging mode, the photoacoustic imaging method can improves greatly the spatial resolution and tissue resolution because the metal elements of the 2D transition metal sulfide nanomaterials have a high atomic number. The $2 \mathrm{D}$ transition metal sulfide nanomaterials have strong absorption ability for $X$ ray. So the $2 \mathrm{D}$ transition metal sulfide nanomaterials can also be used as CT contrast agents. Zhao et al. injected the bidimensional $\mathrm{WS}_{2}$ nanosheet modified by BSA into the nude mice with HeLa tumor, confirm-

ing their good CT imaging effect of $\mathrm{WS}_{2} .{ }^{[29]}$ Yang et al. combined $\mathrm{WS}_{2}$ with $\mathrm{Fe}_{3} \mathrm{O}_{4}$ for MR imaging and used $\mathrm{Cy} 5$ labeled $\mathrm{WS}_{2}-1 \mathrm{O} @ M S-P E G$ for fluorescence imaging. ${ }^{[15]}$ At last, they combined photoacoustic imaging, CT imaging, MR imaging and fluorescence imaging for the multimodality imaging, which provides reliable and comprehensive biological information for the tumor diagnosis (Figure 2). Liu et al. combined the functionalized iron oxide nanoparticles (IONPs) with 2D $\mathrm{MoS}_{2}$ nanosheets forming $\mathrm{MoS}_{2}-\mathrm{IO}$ nanocomposites. ${ }^{[30]}$ Then $64 \mathrm{Cu}$, a commonly used positron-emitting radioisotope, was be adsorbed on the surface of $\mathrm{MoS}_{2}$ without chelating molecule to conduct in vivo positron emission tomography (PET) imaging. Thus, they combined photoacoustic imaging, MR imaging and PET imaging for the multimodality imaging (Figure 3 ).

a

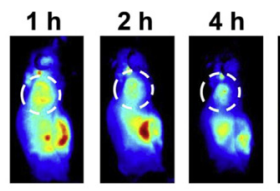

b

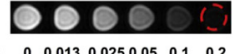

$\begin{array}{llllllllll} & \mathrm{Fe}(\mathrm{mM}) & 0 & 0.013 & 0.025 & 0.05 & 0.1 & 0.2\end{array}$

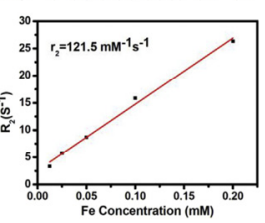

d

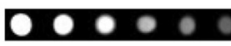

$\underset{\substack{\mathrm{Ws}_{2}-10 @ \mathrm{Mms} \\(\mathrm{mg} / \mathrm{mL})}}{(-2)}$



Figure 2 In vivo multimodal imaging. (a) In vivo fluorescence imaging of 4T1 tumor-bearing mice (b) T2-weighted MR images of $\mathrm{WS}_{2}-\mathrm{IO} @ M S-P E G$ solutions (c) In vivo T2-wighted MR imaged of a mouse (d) CT images and $\mathrm{HU}$ values of $\mathrm{WS}_{2}-\mathrm{IO} @$ MS-PEG solutions with different concentrations. (e) CT images of mice. ${ }^{[15]}$

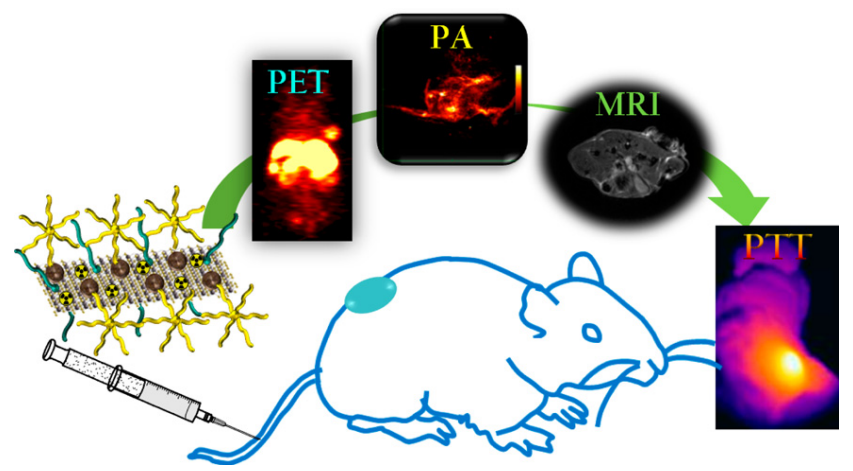

Figure 3 Schematic illustration of the synthesis and application of $\mathrm{MoS}_{2}-\mathrm{IO}$ nanocomposites. ${ }^{[30]}$

\section{Photothermal therapy}

The photothermal therapy is a noninvasive way for treat cancer by using materials that absorb light to generate heat under the light conditions to cause the local high temperatures for killing cancer cells. The photothermal therapy can operate on a selected area, which gets a significant therapeutic effect. 
For example, Qian et al. injected the 2D TiS 2 -PEG nanosheets with a strong NIR absorbance into the mice through the tail vein and found that most of the nanosheets had high retention in tumor areas. ${ }^{[31]}$ They achieved a good therapeutic effect through a laser irradiating tumor areas for the photothermal treatment. Wang et al. proposed an efficient and versatile onepot solvothermal synthesis based on "bottom-up" strategy for the controlled synthesis of PEGylated $\mathrm{MoS}_{2}$ nanosheets with a controlled size, increased crystallinity and excellent colloidal stability. ${ }^{[32]}$ After exposure to $808 \mathrm{~nm}$ laser $\left(1 \mathrm{~W} / \mathrm{cm}^{2}\right)$ for $5 \mathrm{~min}$, 4T1 cells showed a concentration-dependent cell apoptosis implying that $\mathrm{MoS}_{2}$-PEG nanosheets can efficiently transform the NIR light into heat and thus significantly inhibit the in vitro cell proliferation. Li et al. used a hydrothermal method to synthesize the $\mathrm{MoS}_{2}$ nanosheets and then put the vacuum-treating $\mathrm{MoS}_{2}$ nanosheets/soybean phospholipid/chloroform dispersion into a rotary evaporator to synthesize the SP-MoS ${ }_{2}$ nanosheet with an excellent colloidal stability. ${ }^{[33]}$ The obtained SP-MoS nanosheets showed the excellent photothermal stability and conversion performance achieving a good photothermal treatment effect. Zhong et al. used a facile strategy to prepare the PLGA-MoSe $e_{2}$ mixture coated with a folate (FA)-modified PEGlipid shell for the synthesis of multifunctional nanoparticles (FA-PL-MoSe $)_{2}{ }^{[34]}$ The nanoparticles had a minor cytotoxicity, good photothermal conversion capability and great biocompatibility. Additionally, the nanoparticle could target especially to human lung cancer SPCA-1 cells and kill efficiently tumor cells under IR laser (NIR, $808 \mathrm{~nm}$ ) irradiation (Figure 4).

$$
\text { (a) }
$$

(b)

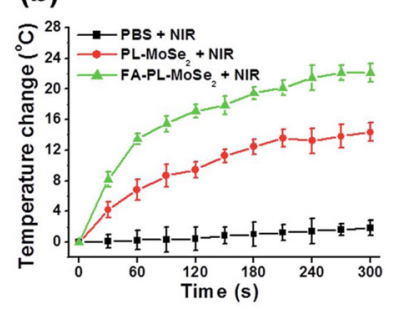

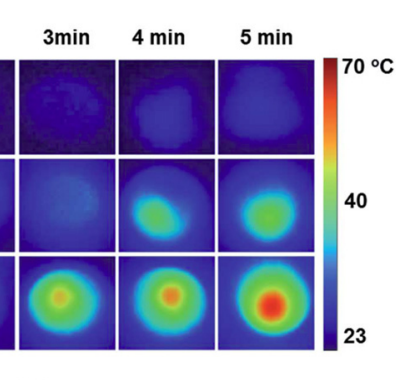

(c)

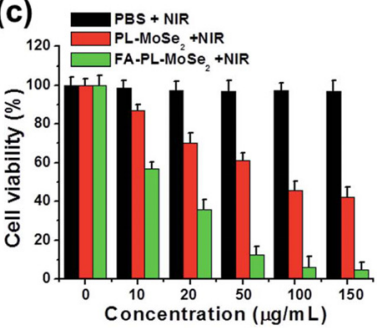

Figure 4 (a) Thermal images of deionized water, PL-MoSe and $\mathrm{FA}-\mathrm{PL}-\mathrm{MoSe}_{2}$, and (b) the corresponding temperature change curves under continuous irradiation with an $808 \mathrm{~nm}$ laser irradiation. (c) Cell viability of SPC-A-1 cells treated with $808 \mathrm{~nm}$ laser irradiation. ${ }^{[34]}$

\section{Photothermal therapy and chemotherapy}

Because 2D transition metal sulfide nanomaterials has a large specific surface area, the researcher always used the nanomaterials to load and convey a series of drug molecules combining the thermal treatment with the chemotherapy so as to treat tumor more fastly and efficiently. Liu et al. modified $\mathrm{MoS}_{2}$ nanosheet with the PEG for a good biocompatibility and then used the $\mathrm{MoS}_{2}$ nanosheets to load DOX. The combined treatment of photothermal therapy and chemotherapy had obtained the excellent synergistic treatment effect. ${ }^{[35]}$ Shao et al. synthesized a novel NIR-light-triggered drug-delivery system by wrapping $\mathrm{MoS}_{2}$ nanosheets around doxorubicin (DOX)-loaded periodic mesoporous organosilicas (PMOs) and then decorated with PEG to form a PMO-DOX@MoS 2 -PEG nanoplatform. ${ }^{[36]}$ The obtained nanoplatforms had a high DOX loading capacity, excellent photothermal transformation ability, and good dispersibility in physiological conditions. Furthermore, the nanoplatforms realized the combined chemotherapy and photothermal therapy for cancer upon NIR laser irradiation. The combined treatment had an improved synergistic therapeutic efficacy (Figure 5).
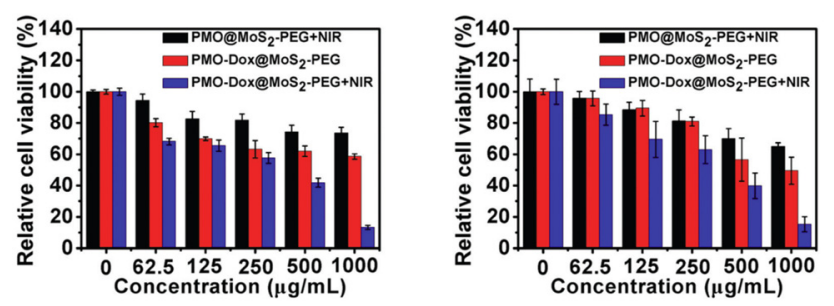

Figure 5 Relative viability of (left) Huh-7 cells and (right) MCF-7 cells treated with PMO@MoS ${ }_{2}-\mathrm{PEG}$ at different PMO concentrations for $4 \mathrm{~h}$ and then incubated for $24 \mathrm{~h}$ after the irradiation with a laser for 5 min and PMO-DOX@MoS 2 -PEG at different PMO concentrations for $4 \mathrm{~h}$, and then incubated for 24 $\mathrm{h}$ after non-irradiation or irradiation with a laser for $5 \mathrm{~min}^{\left[{ }^{[36]}\right.}$

\section{Photothermal and photodynamic therapy}

The photodynamic therapy is a way to kill tumor cells by producing a large amount of reactive oxygen free radicals when the proper laser irradiates the photosensitizer. Compared with chemotherapy and radiotherapy, the photodynamic therapy has relatively minor side effects and a more specific target to kill tumor cells. Liu et al. took advantage of the $\mathrm{MoS}_{2}$ nanosheets' ultra-high surface area to load the photodynamic agent, Chlorin e6 (Ce6), by physical adsorption. ${ }^{[37]}$ In vitro experiments revealed that $\mathrm{Ce} 6$ after being loaded by $\mathrm{MoS}_{2}$-PEG showed remarkably increased cellular uptake and thus significantly enhanced the photodynamic therapeutic efficiency. At the same time, NIR absorbance of the $\mathrm{MoS}_{2}$ nanosheets could enhance photodynamic therapy (Figure 6). Peng et al. used the lipoic acid (LA)-modified PEG and a $\mathrm{pH}$ responsive charge-convertible peptide to modify $\mathrm{MoS}_{2}$ and then loaded a positively charged photosensitizer, toluidine blue $\mathrm{O}$ (TBO) on $\mathrm{MoS}_{2}$ via physical absorption. ${ }^{[38]}$ Because the negatively charged LA-K11 peptide was converted into a positively charged one under acidic conditions, the charge conversion of the peptide could reduce the binding force between positively charged TBO and $\mathrm{MoS}_{2}$, which leaded to the release of TBO. The photo-induced hyperthermia of $\mathrm{MoS}_{2}$ in the tumor areas could also promote TBO release. At the same time, the proper laser irradiated the TBO, which caused the generation of ROS and conducted the photodynamic therapy.

\section{Photothermal therapy and radiotherapy}

The radiotherapy (RT) is an important antitumor modality, which can kill tumor cells through the induction of oxidative stress and/or breaking the DNA of the tumor cells. The RT uses the radiation with various energies to irradiate tumors for inhibiting and killing cancer cells. The RT, however, has its own bottlenecks, for example, the patient was exposed to the high doses of radiation. To improve the radiotherapy effect, scientists used some metal nanoparticles with the strong photoelectric absorption capacity, such as gold nanoparticles ${ }^{[39]}$ or $\mathrm{Bi}_{2} \mathrm{~S}_{3}$ nanoparticles, ${ }^{[40]}$ as radiation dose enhancing agents. Wang et al. utilized the stoichiometric proportion of Mo and $S$ atoms in $\left(\mathrm{NH}_{4}\right)_{2} \mathrm{MoS}_{4}$ to design a reaction with two extra $\mathrm{S}$ atoms and $\mathrm{Bi}$ 
(a)

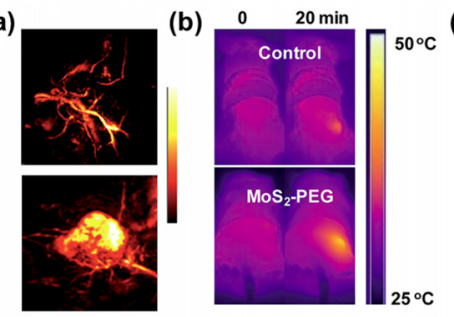

(d)

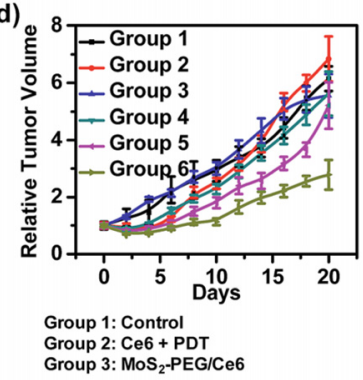

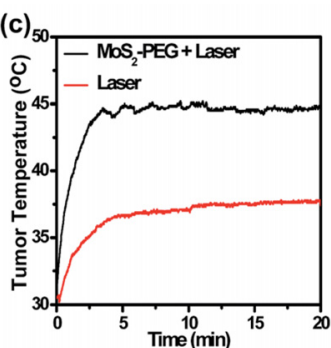

(e)

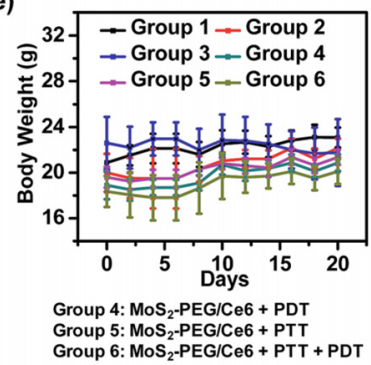

Figure $6 \mathrm{MoS}_{2}-\mathrm{PEG} / \mathrm{Ce} 6$ used for in vivo combination therapy. (a) Photoacoustic and (b) IR thermal images of tumors in mice before and $24 \mathrm{~h}$ after i.v. injection of $\mathrm{MoS}_{2}-\mathrm{PEG} / \mathrm{Ce} 6$. (c) Surface temperature changes of tumors monitored by the IR thermal camera during laser irradiation as indicated in (b). (d) Relative tumor volume curves of different groups of mice after the various treatments indicated. (e) Average body weight of mice after various treatments indicated. ${ }^{[37]}$

atoms during a solvothermal treatment obtaining $\mathrm{MoS}_{2} / \mathrm{Bi}_{2} \mathrm{~S}_{3}-$ PEG (MBP) composite nanosheets. ${ }^{[41]}$ The $\mathrm{Bi}$ atoms of MBP composite nanosheets have a strong photoelectric absorbance capacity under $\mathrm{X}$-ray radiation and can generate numerous short-range secondary electrons. Therefore, MBP composite nanosheets show a dose-enhanced capability in tumor radiotherapy. At the same time, the $\mathrm{MoS}_{2}$ framework endowed the MBP nanosheets with an excellent photothermal transformation capacity. Such a MBP composite nanosystem was able to realize photothermal therapy and radiotherapy, which was treated for tumor and achieved good therapeutic effect (Figure 7).

\section{Conclusions}

Although they have poor water solubility and bio-compatibility, 2D nanomaterials have many excellent properties such as the large surface area, more binding sites and excellent photothermal properties. In order to make 2D nanomaterials apply in the field of medicine, many reports use biological molecules to modify, which make them have a good hydrophilicity and bio-compatibility and realize the $2 \mathrm{D}$ material metabolize in the body reducing its toxicity in organism. Meanwhile, the modified 2D nanomaterials are used to load drugs for photothermal treatment and chemotherapy. Furthermore, the 2D nanomaterials can also be assembled with other functional nanomaterials, or added other elements for realizing their multi-functional applications in the field of the biomedical science.

\section{Acknowledgement}

The work is supported by the Research Project of the Shanghai Municipal Science and Technology Commission (No. 15441907000).



Figure 7 Schematic illustration of solvothermal synthesis of MBP nanosheets and tumor PTT and RT. ${ }^{[41]}$

\section{References}

[1] (a) Novoselov, K.; Geim, A. K.; Morozov, S.; Jiang, D.; Katsnelson, M.; Grigorieva, I.; Dubonos, S.; Firsov, A. Nature 2005, 438, 197; (b) Sun, Y.; Gao, S.; Lei, F.; Xiao, C.; Xie, Y. Acc. Chem. Res. 2015, 48, 3.

[2] Butler, S. Z.; Hollen, S. M.; Cao, L.; Cui, Y; Gupta, J. A.; Gutiérrez, H. R.; Heinz, T. F; Hong, S. S.; Huang, J; Ismach, A. F.; Johnston-Halperin, E.; Kuno, M.; Plashnitsa, V. V.; Robinson, R. D.; Ruoff, R. S.; Salahuddin, S.; Shan, J.; Shi, L.; Spencer, M. G.; Terrones, M. ACS Nano 2013, 7, 2898.

[3] Behzad, S. J. Solid State Commun. 2016, 248, 27

[4] Wang, Z. G.; Su, Q. L.; Yin, G. Q.; Shi, J. J.; Deng, H. Q.; Guan, J.; Wu, M. P.; Zhou, Y. L.; Lou, H. L.; Fu, Y. Q. J. Mater. Chem. Phys. 2014, 147, 1068.

[5] Poizot, P.; Laruelle, S.; Grugeon, S.; Dupont, L.; Tarascon, J. M. Nature 2000, 407, 496

[6] Anasori, B.; Lukatskaya, M. R.; Gogotsi, Y. Nat. Rev. Mater. 2017 2, 16098.

[7] Song, J.; Wang, J.; Lin, X. Y.; He, J. F.; Liu, H. L.; Lei, Y. P.; Chu, Z. Y. ChemElectroChem 2017, 4, 2373.

[8] Eda, G.; Yamaguchi, H.; Voiry, D.; Fujita, T.; Chen, M.; Chhowalla, M. Nano Lett. 2011, 11, 5111.

[9] Bang, G. S.; Nam, K. W.; Kim, J. Y.; Shin, J.; Choi, J. W.; Choi, S. Y. ACS Appl. Mater. Interfaces 2014, 6, 7084.

[10] Chen, Y.; Tan, C.; Zhang, H.; Wang, L. Chem. Soc. Rev. 2015, 44, 2681.

[11] Radisavljevic, B.; Radenovic, A.; Brivio, J.; Giacometti, V.; Kis, A. J. Nat. Nanotechnol. 2011, 6, 147.

[12] Yin, Z.; Li, H.; Li, H.; Jiang, L.; Shi, Y.; Sun, Y.; Lu, G.; Zhang, Q.; Chen, X.; Zhang, H. ACS Nano 2011, 6, 74.

[13] Novoselov, K.; Jiang, D.; Schedin, F.; Booth, T.; Khotkevich, V.; Morozov, S.; Geim, A. J. Proc. Natl. Acad. Sci. U. S. A. 2005, 102, 10451.

[14] Zeng, Z. Y.; Yin, Z. Y.; Huang, X.; Li, H.; He, Q. Y.; Lu, G.; Boey, F.; Zhang, H. Angew. Chem. Int. Ed. 2011, 50, 11093.

[15] Yang, G. B.; Gong, H.; Liu, T.; Sun, X. Q.; Cheng, L.; Liu, Z. Biomaterials 2015, 60, 62 .

[16] Coleman, J. N.; Lotya, M.; O'Neill, A.; Bergin, S. D.; King, P. J; 
Khan, U.; Young, K.; Gaucher, A.; De, S.; Smith, R. J.; Shvets, I. V.; Arora, S. K.; Stanton, G.; Kim, H. Y.; Lee, K.; Kim, G. T.; Duesberg, G. S.; Hallam, T.; Boland, J. J.; Wang, J. J.; Donegan, J. F.; Grunlan, J. C.; Moriarty, G.; Shmeliov, A.; Nicholls, R. J.; Perkins, J. M.; Grieveson, E. M.; Theuwissen, K.; McComb, D, W.; Nellist, P. D.; Nicolosi, V. Science 2011, 331, 568.

[17] Smith, R. J.; King, P. J.; Lotya, M.; Wirtz, C.; Khan, U.; De, S.; O'Neill, A.; Duesberg, G. S.; Grunlan, J. C.; Moriarty, G.; Chen, J.; Wang, J.; Minett, A. I.; Nicolosi, V.; Coleman. J. N. Adv. Mater. 2011, 23, 3944.

[18] Elías, A. L.; Perea-López, N.; Castro-Beltrán, A.; Berkdemir, A.; Lv, R. T.; Feng, S. M.; Long, A. D.; Hayashi, T.; Kim, Y. A.; Endo, M.; Gutiérrez, H. R.; Pradhan, N. R.; Balicas, L.; Mallouk, T. E.; López-Urías, F.; Terrones, H.; Terrones, M. ACS Nano 2013, 7, 5235.

[19] Najmaei, S.; Liu, Z.; Zhou, W.; Zou, X. L.; Shi, G.; Lei, S. D.; Yak-obson, B. I.; Idrobo, J. C.; Ajayan, P. M.; Lou. Nat. Mater. 2013, 12, 754.

[20] Lee, Y. H.; Zhang, X. Q.; Zhang, W. J.; Chang, M. T.; Lin, C. T.; Chang, K. D.; Yu, Y. C.; Wang, J. T. W.; Chang, C. S.; Li, L. J.; Lin, T. W. Adv. Mater. 2012, 24, 2320.

[21] Xu, G. C.; Lu, Z. X.; Zhang, Q.; Qiu, H. L.; Jiao, L. Y. Acta Chim. Sinica 2015, 73, 895

[22] Seo, J. W.; Jun, Y. W.; Park, S. W.; Nah, H.; Moon, T.; Park, B.; Kim, J. G.; Kim, Y. J.; Cheon, J. Angew. Chem. Int. Ed. 2007, 46, 8828.

[23] Jeong, S.; Yoo, D.; Jang, J. T.; Kim, M.; Cheon, J. J. Am. Chem. Soc. 2012, 134, 18233.

[24] Wang, Y.; Ni, Y. Anal. Chem. 2014, 86, 7463.

[25] Ren, X. P.; Pang, L. Q.; Zhang, Y. X.;Ren, X. D.; Fan, H. B.; Liu, S. Z. J. Mater. Chem. 2015, 3, 10693.

[26] Liu, Q.; Sun, C. Y.; He, Q.; Khalil, A.; Xiang, T.; Liu, D. B.; Zhou, Y.; Wang, J.; Song, L. Nano Res. 2015, 8, 3982.

[27] Wang, L. V.; Hu, S. Science 2012, 335, 1458

[28] Qian, X. X.; Shen, S. D.; Liu, T.; Cheng, L.; Liu, Z. Nanoscale 2015,

\section{$7,6380$.}

[29] Yong, Y.; Zhou, L.; Gu, Z.; Yan, L.; Tian, G.; Zheng, X.; Liu, X.; Zhang, X.; Shi. J.; Cong, W. Nanoscale 2014, 6, 10394.

[30] Liu, T.; Shi, S. X.; Liang, C.; Shen, S. D.; Cheng, L.; Wang, C.; Song, X. J.; Goel, S. Y.; Barnhart, T. E.; Cai, W. B.; Liu, Z. ACS Nano 2015, 9, 950.

[31] Qian, X, X.; Shen, S. D.; Liu, T.; Cheng, L.; Liu, Z. Nanoscale 2015, 7,6380 .

[32] Wang, S. G.; Li, K.; Chen, Y.; Chen, H. G.; Ma, M.; Feng, J. W.; Zhao, Q. H.; Shi, J. L. Biomaterials 2015, 39, 206.

[33] Li, X.; Gong, Y.; Zhou, X. Q.; Jin, H.; Yan, H. H.; Wang, S. G.; Li, J. Int. J. Nanomed. 2016, 11, 1819.

[34] Zhong, C. L.; Zhao, X.; Wang, L. J.; Li, Y. X.; Zhao, Y. Y. RSC Adv. 2017, 7, 7382 .

[35] Liu, T.; Wang, C.; Gu, X.; Gong, H.; Cheng, L.; Shi, X. Z.; Feng, L. Z.; Sun, B. Q.; Liu, Z. Adv. Mater. 2014, 26, 3433.

[36] Shao, T. P.; Wen, J.; Zhang, Q.; Zhou, Y. W.; Liu, L.; Li, H.; Yu, W.; Tian, Y.; Zhang, Y. L.; Tian, W.; Su, Y. Y.; Teng, Z. G.; Lu, G. M.; Xu, J. J. Mater. Chem. B 2016, 4, 7708.

[37] Liu, T.; Wang, C.; Cui, W.; Gong, H.; Liang, C.; Shi, X. Z.; Li, Z. W.; Sun, B. Q.; Liu, Z. Nanoscale 2014, 6, 11219.

[38] Peng, M. Y.; Zheng, D. W.; Wang, S. B.; Cheng, S. X.; Zhang, X. Z. ACS Appl. Mater. Interfaces 2017, 9, 13965.

[39] Chithrani, D. B.; Jelveh, S.; Jalali, F.; Prooijen, M. V.; C. Allen, C.; Bristow, R. G.; Hill, R. P.; Jaffray, D. A. Radiat. Res. 2010, 173, 719.

[40] Ma, M.; Huang, Y.; Chen, H.; Jia, X.; Wang, S.; Wang, Z.; Shi, J. Biomaterials 2015, 37, 447.

[41] Wang, S. G.; Li, X.; Chen, Y.; Cai, X. J.; Yao, H. L.; Gao, W.; Zheng, Y. Y.; An, X.; Shi, J. L.; Chen, H. G. Adv. Mater. 2015, 6, 2775.

Received June 25, 2018 Accepted June 26, 2018 